\title{
SCEPTICISME ET POLITIQUE
}

\author{
Catherine LARRERE
}

RÉsumÉ : La dimension politique du scepticisme, considère-t-on souvent, c'est le conformisme. Mais la séparation entre le domaine intérieur et l'espace public n'a pas pour seule conséquence l'injonction de suivre la coutume. Elle est d'abord constitution du domaine privé, qui est un lieu de liberté, à l'écart de la foule et du pouvoir. Cela permet de mieux comprendre la position sceptique en politique : le conseil du prince, en secret et en retrait de l'éclat public. Cela permet surtout de comprendre ce qu'apporte le scepticisme aux conceptions modernes de la liberté : un modèle social de la libre poursuite de la vérité.

Mots-Clés : scepticisme, liberté politique, séparation du privé et du public, modèles sociaux de la science.

ABSTRACT : Scepticism is generally considered as leading to political conformity. But obedience to custom is not the only consequence of the separation between the private and the public spheres. The main result of this separation is to create an area of private life which is a space of freedom, away from the crowd and the authorities. It is thus easier to understand the sceptical stance in politics, advising the Prince secretly, while keeping out of the limelight. Above all, this helps us to appreciate the contribution of scepticism to modern conceptions of liberty in providing a social model for the free pursuit of truth.

KEYWORDS : scepticism, political liberty, separation of public and private lives, social models of knowledge.

Revue de synthèse : $4^{\mathrm{e}}$ S. $\mathrm{n}^{\mathrm{os}}$ 2-3, avr.-sept. 1998, p. 271-292. 
ZuSAMMENFASSUNG : Nach einer verbreiteten Ansicht führt der Skeptizismus zu politischem Konformismus. Aber die Befolgung vorgegebener Normen ist nicht die einzige Konsequenz, die sich aus der Trennung von Privatsphäre und öffentlicher Sphäre ergibt. Das wichtigste Ergebnis dieser Trennung ist die Bildung eines privaten Bereichs als Ort der Freiheit, der dem Einfluß der Massen und der Autoritäten entzogen ist. Diese Erkenntnis führt zu einem besseren Verständnis der skeptischen Haltung in der Politik: Man muß den Herrscher im Stillen beraten, ohne öffentliches Aufsehen zu erregen. So wird auch der Beitrag des Skeptizismus zum modernen Freiheitsbegriff deutlich : Er liefert ein soziales Modell bei der Suche nach der Wahrheit.

STICHWÖRTER : Skeptizismus, politische Freiheit, Trennung von Privatleben und Öffentlichkeit, soziale Wissenschaftsmodelle.

Catherine LARRÈRE, née en 1944, est professeur de philosophie à l'université de Bordeaux III. Spécialiste de philosophie politique, elle s'intéresse également aux problèmes liés à la nature et à l'environnement.

Adresse : UFR de philosophie, Université Michel de Montaigne-Bordeaux III, Domaine universitaire, F-33405 Talence Cedex.

Courrier électronique : clarrer@worldnet.fr 
En montrant que le scepticisme, jusqu'à Spinoza, pouvait s'interpréter comme un fidéisme, Richard H. Popkin a insisté sur la signification religieuse de ce moment de l'histoire intellectuelle de l'époque moderne. Il s'agit ici d'en faire apparaître la dimension politique. Une des idées directrices du livre de Popkin - et qu'il a clairement énoncée dans les présentations ultérieures qu'il en a faites - est que ce n'est pas simple coïncidence si les Hypotyposes pyrrhoniennes de Sextus Empiricus, qui venaient d'être rééditées, ont été utilisées dans la querelle religieuse. Le pyrrhonisme a été délibérément enrôlé au service de l'Église : c'est à l'initiative d'« un éminent catholique français, Gentien Hervet, secrétaire du cardinal de Lorraine », que Sextus Empiricus fut publié en latin en $1569^{\prime}$. L'histoire du scepticisme est alors celle de l'alliance, pendant "les soixante-quinze années qui suivirent le concile de Trente », entre la Contre-Réforme ${ }^{2}$ et les nouveaux pyrrhoniens, une alliance dont l'objectif était d'annihiler la force intellectuelle qu'était devenu le calvinisme en France. Popkin insiste sur la réalité vécue de cette alliance en parlant de «l'amitié personnelle » et des « sentiments mutuels d'admiration » qui existaient entre des membres du clergé catholique et certains sceptiques.

C'est donc contre un même ennemi dogmatique que s'est faite l'unité entre clercs et sceptiques, plus précisément ce groupe d'intellectuels qui est au centre du dispositif argumentatif de Popkin : les libertins érudits.

«Il n'est pas du tout impossible, avance ainsi Popkin, que ce que l'on nomme libertinage érudit n'ait cherché ni à détruire, ni à ébranler le christianisme, mais à servir de support à un certain type de catholicisme libéral opposé tant à la croyance superstitieuse qu' au protestantisme fanatique ${ }^{3}$. ”

La thèse religieuse du fidéisme a bien un contrepoint politique, celle d'un « catholicisme libéral », qui vient compléter et renforcer la dimension religieuse. À l'intérêt qu'avait l'Église catholique à s'appuyer sur le scepticisme pour défendre la foi, correspond, symétriquement, l'intérêt qu'avaient les sceptiques à accepter des positions fidéistes pour garantir leur propre liberté : «Rien n'empêchait probablement que des libéraux de

1. Richard H. Popkin, Histoire du scepticisme d'Érasme à Spinoza, trad. Christine Hivet, présentation Catherine LARRère, Paris, Presses universitaires de France (Léviathan), 1995 (cité par la suite comme $H S$ ), p. 111.

2. On parle plutôt, ces derniers temps, de Réforme catholique, mais dans l'optique de Popkin, l'expression de Contre-Réforme convient mieux.

3. $H S$, p. 143. 
diverses tendances se sentissent plus à l'aise à l'intérieur de l'Église que dans le monde dogmatique des réformateurs ${ }^{4}$. $\gg$ Dans le fidéisme catholique, les sceptiques pouvaient en effet trouver, selon Popkin, une « religion plus simple», le "noyau du christianisme», une attitude plus «morale que religieuse», ce qui leur convenait mieux qu'un intégrisme dogmatique, et leur permettait de se maintenir à distance de la superstition populaire sans attaquer directement le catholicisme. Dans ce catholicisme tolérant, à la fois minimaliste ${ }^{5}$ et pluraliste, les sceptiques, selon Popkin, pouvaient trouver leur place.

Cette idée d'un « catholicisme libéral » a retenu mon attention. Elle présente une vision de la Contre-Réforme à laquelle nous ne sommes guère habitués, et, surtout, elle permet de saisir la dimension politique de l'interprétation non conformiste que fait Popkin du scepticisme. Des « libertins érudits », il fait des « libéraux » : leur filiation n'est pas celle des « esprits forts », des matérialistes dogmatiques, des athées, du petit père Combe et du militantisme antireligieux, mais celle d'un catholicisme tolérant, où s'esquisse une généalogie «à l'anglaise » d'un libéralisme français qu'il reste à définir. C'est dans cet esprit que, dans ma présentation de la traduction de Popkin, il était proposé de voir dans le scepticisme une source originale de l'individualisme libéral, qui s'ajouterait à l'opposition classique des deux principaux paradigmes, ceux de l'humanisme civique et du droit naturel moderne. Chacun de ces deux paradigmes organise un espace: l'espace politique, la sphère des échanges. Le scepticisme se place ailleurs. À l'opposé de l'humanisme civique, le modèle sceptique est celui d'une vie à l'écart du monde politique, il ne fait pas du vivere civile un devoir moral. Il ne fait pas non plus une théorie universaliste de l'égale liberté, à la façon du droit naturel moderne. Cela expose ce scepticisme libéral (s'il existe) à l'élitisme, mais cela lui permet de poser un lieu nouveau, original, de la liberté : non pas la sphère des échanges (la sociabilité du droit naturel moderne), mais le domaine privé, l'intimité de la maison. On peut ainsi se demander si Montesquieu, ce président bordelais, qui, comme Montaigne, renonça aux charges publiques, pour vivre à l'écart, n'a pas trouvé, dans la

4. $H S$, p. 143.

5. Comme le fait remarquer Jacqueline LAGRÉE, dans ce même volume, p. 257-269, dans son article intitulé "Religion naturelle et scepticisme religieux ", Popkin n'emploie pas l'expression de religion naturelle. Mais cette idée d'une "religion plus simple », dont on ne garderait que le noyau, correspond bien au minimalisme caractéristique de la religion naturelle. Peut-être alors faudrait-il chercher à celle-ci également des origines sceptiques, et pas seulement stoïciennes, à la façon dont Richard Tuck a pu montrer que le minimalisme du droit naturel moderne est une réponse au scepticisme. Voir Richard Tuck, « The "modern" theory of natural law ", in The Languages of political theory in early modern Europe, éd. Anthony PAdgen, Cambridge, Cambridge University Press, 1986, p. 99-122. 
tradition sceptique, l'idée que la sûreté a un lieu, qui est l'espace domestique : "Quand un homme est fidèle aux lois, il a satisfait à ce qu'il doit au prince. Il faut au moins qu'il ait sa maison pour asile, et le reste de sa conduite en sûreté ${ }^{6}$."

Que l'espace privé puisse être, à l'époque moderne, le lieu d'une affirmation forte de l'individualisme, c'est ce dont j'ai, depuis, trouvé une confirmation savante dans un article d'Ivo Comparato, "Un exemple d'individualisme moderne. La politique et le malaise des intellectuels à l'âge baroque ${ }^{7}$ ». Comparato y montre en effet comment le domaine privé devient le lieu où s'affirme l'individualisme des humanistes lettrés, lorsque s'efface la conjoncture qui avait non seulement permis l'humanisme civique, républicain, mais également ouvert la cour du Prince aux intellectuels.

Mon but est donc, en repartant de cet article, d'argumenter la thèse, trouvée chez Popkin, d'une contribution sceptique à l'individualisme libéral moderne. Cela suppose trois choses. Premièrement, de montrer qu'il y a bien un apport du scepticisme à cette «privatisation» de la liberté qui marque, selon Comparato, le passage de la Renaissance à l'âge baroque. Deuxièmement, il faut pouvoir éclairer ce sur quoi bute généralement l'analyse politique du scepticisme moderne : la participation des sceptiques, et tout particulièrement des libertins érudits, ces conseillers du prince, à la mise en place de l'absolutisme. Cela ne remet-il pas en cause aussi bien l'association du libéralisme et du scepticisme, que l'affirmation de la dimension privée de leur conception de la liberté ? Troisièmement, et enfin, cela devrait permettre de mettre à l'épreuve la thèse de Popkin, celle d'un intérêt commun des intellectuels sceptiques et des clercs catholiques à s'opposer au dogmatisme calviniste. Elle se heurte en effet à une idée reçue, qui met la liberté de conscience, revendiquée par la Réforme protestante, à l'origine de la liberté d'expression. Quel modèle de liberté le scepticisme avait-il donc à offrir?

6. Montesquieu, De l'esprit des lois, Genève, 1748, liv. XII, chap. XXII, Paris, Garnier frères, 1973, t. 1, p. 223. On remarquera que pour considérer, comme on le fait généralement, la fin du Tartuffe de Molière comme un dénouement heureux plaqué sur une histoire prête à mal finir, il faut admettre la supposition contraire : rien n'échappe à l'œil du prince, pas même l'intimité familiale, et ses envoyés pénètrent partout. C'est la même démarche qui conduisait les sujets du roi à lui demander des lettres de cachet pour résoudre leurs problèmes familiaux, comme l'ont montré Michel Foucault et Arlette FARGE, in Le Désordre des familles. Lettres de cachet des archives de la Bastille, Paris, Gallimard, 1982. L'inviolabilité du privé n'est pas un vou unanime à la fin de l'Ancien Régime.

7. Ivo Comparato, « Un exemple d'individualisme moderne. La politique et le malaise des intellectuels à l'Âge baroque », in L'Individu dans la théorie politique et dans la pratique, sous la dir. de Janet Coleman, Paris, Presses universitaires de France, 1996, chap. vil, p. 173196. 


\section{I. - INDIVIDUALISME MODERNE ET SCEPTICISME}

Les rapports entre les intellectuels et le pouvoir se sont renforcés pendant la Renaissance, selon Comparato. Ce fut d'abord le retour de l'humanisme civique, mis en évidence par les travaux de Hans Baron ${ }^{8}$, l'éloge du vivere civile, de la supériorité de la vita activa sur la vita contemplativa. À Florence, les lettrés ont mis leur savoir classique au service de la République, et l'on a considéré que les humanités étaient la formation qui convenait aux hommes politiques ${ }^{9}$. Mais le mouvement débordait le cadre républicain, les cours princières attiraient les humanistes, orientant l'activité intellectuelle vers le domaine politique.

À la fin du $\mathrm{XVI}^{\mathrm{e}}$ siècle, ce rapprochement est terminé. Ce ne sont pas les humanistes qui ont quitté le pouvoir, c'est plutôt celui-ci qui n'a plus eu besoin de leurs services. La rationalisation du pouvoir, la mise en place de l'absolutisme ont conduit à des modes de spécialisation des fonctions politiques. On s'est mis à avoir surtout besoin de juristes et les humanistes polyvalents se sont retrouvés sans emploi. C'est le moment de leur retrait du domaine public. Cela les conduit à créer un espace social et intellectuel distinct et indépendant des institutions publiques : les académies, nouveau mode d'organisation intellectuelle, apparaissent alors, et leur succès, selon Comparato, fut «si grand en Italie que l'on passa de 12 académies en 1530 à plus de 370 vers la fín du siècle ${ }^{10}$ ». En même temps, on voit apparaître, chez ces intellectuels, de nouvelles formes d'attention à soi, un intérêt pour l'identité personnelle, pour le «je», une recherche de l'intériorité. «L'identité est au cœur du problème, explique Comparato, _ l'identité de ceux qui ne jouissent pas de la sérénité qu'apporte le fait de ressembler à quelque chose, ou de ceux à qui cela ne saurait suffire ${ }^{1 !}$. »

Si l'on rapproche cette caractéristique de la précédente (la formation d'espaces sociaux consacrés à l'esprit), on se rend compte que l'attention à soi, le goût de l'intériorité ne sont nullement synonymes de solitude, mais s'affirment dans un espace commun, où des rencontres sont possibles.

8. Hans BARON, The Crisis of the early Italian Renaissance. Civic humanism and republican liberty in an age of clacissism and tyranny, éd. rev., Princeton. Princeton University Press, 1966. John Greville Agard Pocock, The Machiavellian Moment. Florentine political thought and the Atlantic republican tradition. Princeton, Princeton University Press, 1975.

9. Quentin Skinner, Machiavel, postf. et trad. de l'anglais Michèle Plon, Paris, Seuil, 1989.

10. I. Comparato, art. cit. supra n. 7, p. 176.

11. Ibid., p. 180. 
D'où l'indiscutable élitisme d'une telle retraite : on se met à l'écart du monde, ce théâtre d'apparences, pour goûter, entre esprits choisis, les plaisirs de conversations à l'antique. La forte affirmation de soi à laquelle conduit cette quête d'identité, se fait donc dans un espace, qui, tout en étant privé, séparé du monde extérieur, n'en est pas moins un espace social de communication $^{12}$.

En cherchant quels furent les modèles antiques d'un tel individualisme, Comparato montre l'importance du stoïcisme, tardivement relayé, selon lui, par l'épicurisme. Ce n'est qu'avec François de La Mothe Le Vayer, et ses Dialogues faits à l'imitation des Anciens, qu'il en vient à évoquer un apport proprement sceptique à ce courant individualiste. La critique des certitudes politiques, à laquelle il se livre dans «De la politique », le conduirait à mettre le code des sceptiques antiques au service d'une exaltation de l'individu contre l'État. Cependant, l'importance que donne Comparato, dans sa présentation de l'affirmation individualiste, à Montaigne, tout comme les références qu'il fait à Pierre Charron, montrent que la contribution sceptique est sans doute plus précoce et plus centrale. On peut même montrer que deux des caractéristiques principales de cet individualisme moderne (la subjectivité et la liberté) proviennent du courant sceptique, non de la reprise de ses arguments antiques, mais de la contribution proprement moderne de Montaigne et de Charron, au scepticisme.

Il y a d'abord la subjectivité, cette forme moderne d'affirmation de l'individualisme. Or si Montaigne en est, sans conteste, le principal introducteur, c'est bien qu'il n'a pas besoin du donné stable d'une nature pour affirmer l'authenticité et la sincérité de son rapport à lui-même, mais qu'il est pris dans une quête sans fin, un perpétuel mouvement d'échappement à soi-même, de passage, dans lequel se reconnaît le sujet, « cette pâle transition qui donne sa couleur aux différents gestes de notre vie ${ }^{13} »$. Il n'y a donc pas moi et les autres, mais un constant glissement du « je » qui reconduit vers les autres. La « solitude », pour Montaigne, c'est se mettre à l'écart des devoirs, des « offices », du vivere civile. Cela n'implique nullement d'ignorer les hommes, si ce sont des amis :

«La solitude que j'aime et que je prêche, ce n'est principalement que ramener à moi mes affections et mes pensées, restreindre et resserrer non mes pas, ains mes désirs et mon souci, résignant la sollicitude étrangère et fuyant mortelle-

12. Jürgen Habermas a bien montré comment chaque sphère, y compris la sphère privée, a sa dimension publique. Voir Jürgen HaBermas, L'Espace public. Archéologie de la publicité comme dimension constitutive de la société bourgeoise, trad. de l'allemand par Marc B. DE Launay, Paris, Payot, 1978.

13. Jocelyn BENOIST, «Subjectivité », in Notions de philosophie, vol. II, sous la dir. de Denis Kambouchner, Paris, Gallimard (Folio/Essais, 278), 1995, p. 555. 
ment la servitude et l'obligation, et non tant la foule des hommes, que la foule des affaires ${ }^{14}$."

La « liberté et jurisdiction de voir, juger, examiner toutes choses ${ }^{15}$ » est une revendication constante : Charron loue « la pleine, entière, généreuse et seigneuriale liberté d'esprit ${ }^{16} »$. On peut y voir l'application du principe sceptique de la suspension de jugement, de l'epoché. Celle-ci, dans le scepticisme antique, mène à l'ataraxie, car elle permet de ne plus être troublé par les opinions, comme Montaigne le rappelle ainsi fort clairement:

« [...] cette assiette de leur jugement, droite et inflexible, recevant tous objets sans application et consentement, les achemine à leur ataraxie, qui est une condition de vie paisible, rassise, exempte des agitations que nous recevons par l'impression de l'opinion et science que nous pensons avoir des choses ${ }^{17}$."

Mais sa reprise moderne n'incline pas la pratique de l'epoché vers l'indifférence, elle la reconduirait plutôt vers l'incessante recherche d'une vérité qui nous échappe, en "un dessein enquêrant plutôt qu'instruisant ", comme dit Montaigne ${ }^{18}$. Le scepticisme n'est pas une position, il est le mouvement même de l'esprit, la quête sans fin de la vérité. Cette quête se fait en commun : «J'aime, entre les galants hommes, qu'on s'exprime courageusement, que les mots aillent où va la pensée ", remarque Montaigne, qui ajoute que la pratique de l'epoché incline à la tolérance : « [...] nous autres qui privons notre jugement du droit de faire des arrêts regardons mollement les opinions diverses ${ }^{19}$. »

Des lettrés discutant à loisir, paisiblement, à l'écart du monde, des sujets de leur choix : c'était déjà le cas des diverses écoles philosophiques de l'Antiquité, de la période hellénistique, plus particulièrement. La disparition de la cité, à laquelle se substitue la domination impériale, conduit à un renforcement de l'individualisme. Participer à la vie politique n'est plus un mode d'accomplissement de la vie heureuse. C'est à l'écart de la ville et de ses lieux de pouvoir que se réunissent stoïciens ou épicuriens. Montaigne rejette cependant fermement le dogmatisme de ces derniers qui ne lui sont nullement un modèle; et «l'entre-soi » qu'il préconise entre amis n'a pas

14. Michel DE Montaigne, Essais, liv. III, chap. III, «Des trois commerces", in Euvres complètes, Paris, Gallimard (Bibliothèque de la Pléiade), 1962, p. 801.

15. Pierre Charron, De la sagesse, 1601-1604, préf., $2^{e}$ éd., Paris, Fayard (Corpus des œuvres de philosophie en langue française), 1986, p. 34.

16. Ibid., liv. II, chap. II, p. 385.

17. Essais, liv. II, chap. XII, « Apologie de Raymond Sebond », in op. cit. supra n. 14, p. 483 .

18. Ibid., p. 489.

19. Essais, liv. III, chap. viII, « De l'art de conférer », in op. cit. supra n. 14, p. 901. 
la rigidité communautaire de la secte philosophique. $\mathrm{Si}$, comme le reconnaît Comparato, il décrit si bien la façon dont le domaine privé se sépare de l'espace public ${ }^{20}$, cela vient de ce que cette séparation s'accomplit dans sa démarche même - qui est une démarche sceptique. C'est à la fin du chapitre sur la coutume, où il reprend, avec allégresse, tous les arguments du relativisme sceptique, que Montaigne prononce la séparation entre «la juridiction privée », lieu de la liberté, et le domaine public, lieu de l'obéissance : «Car c'est la règle des règles et générale loi des lois, que chacun observe celle du lieu où il est ${ }^{21}$. » «Intus ut libet, foris ut moris $e s t^{22} »:$ la dualité sceptique, en même temps qu'elle organise un espace intérieur, règle le rapport à la politique.

\section{II. - SCEPTICISME ET POLITIQUE}

Selon Michel Villey, le scepticisme conduit nécessairement au positivisme juridique ${ }^{23}$. Montaigne remarque en effet que "les lois se maintiennent en crédit, non parce qu'elles sont justes, mais parce qu'elles sont lois ${ }^{24} »$. Le positivisme juridique, comme le fidéisme, n'est-il pas une façon d'accepter les choses sans s'interroger sur leur raison d'être? On passerait ainsi aisément de Montaigne à Hobbes - pour qui c'est l'autorité, non la raison, qui fait la loi. On peut ainsi parler d'un accord passif entre scepticisme et absolutisme. Montaigne fuit les «divisions séditieuses et querelleuses ", cherche la tranquillité : si l'obéissance est le prix de la paix, alors on obéira. On retrouve bien l'idée de Hobbes : c'est dans sa capacité à assurer la paix civile que l'absolutisme prouve sa validité. La liberté des sujets, alors, s'accommode d'un pouvoir fort.

Mais passer de Montaigne à Hobbes, c'est voir se développer une science du politique, dont l'exposition est, à elle seule, un acte politique :

20. Voir I. Comparato, art. cit. supra, n. 7, p. 186 : «Pour décrire l'acte qui sépare ce domaine du monde extérieur, le justifie et lui donne toute sa densité, rien n'a été écrit de plus pénétrant que les Essais de Montaigne. "

21. Essais, liv. I, chap. XXIII, « De la coutume et de ne changer aisément une loi reçue », in op. cit. supra n. 14, p. 117.

22. Voir Guy Patin, Lettre à mon fils, in Antoine Adam, Les Libertins au xvIr siècle, Paris, Buchet-Chastel, 1964, p. 157 : « À l'intérieur, comme il plaît, à l'extérieur, selon la coutume. » La formule y est présentée comme « la maxime des Italiens ».

23. Michel VILLEY, La Formation de la pensée juridique moderne, Paris, Montchrestien, 1968, p. 488 : «Le fruit du scepticisme dans le droit, ce sera le positivisme, au sens le plus complet du mot. "Villey, dont le père édita Montaigne, partage le jugement de Popkin sur celui-ci, voir ibid., p. 489: «Nous savons tous que chez Montaigne, précurseur des Pensées de Pascal, le scepticisme sert de moyen pour justifier la foi chrétienne."

24. Essais, liv. III, chap. XIII, « De l'expérience », in op. cit. supra n. 14, p. 1049. 
aussi doit-elle être autorisée par le pouvoir. Rien de tel chez Montaigne. «La raison privée n'a qu'une jurisdiction privée ${ }^{25}$ », et loin de lui l'idée de développer quelque savoir politique que ce soit. La seule attitude cohérente, semble-t-il, c'est l'abstention. Pour Charron, le seul « esprit universel » est la « générale indifférence ${ }^{26}$ qui justifie le retrait a-politique hors du monde public : «Nous avons tous assez d'affaire chez et au-dedans de nous, sans s'aller perdre au-dehors et se donner à tous : il se faut tenir à soimême ${ }^{27}$." La dualité sceptique (du dedans et du dehors, de la liberté de pensée et du conformisme des conduites) se prolonge dans l'opposition du sage et du populaire : le sage est celui qui se tient à l'écart de la foule, de ses emportements passionnels et de ses opinions inconsistantes.

Cette indifférence va de pair avec ce que l'on désigne comme le conservatisme $^{28}$, ou le conformisme, sceptique : Montaigne déconseille « de ne changer aisément une loi reçue ». Sans doute Hobbes affirme-t-il que celui qui entreprend de réformer l'État s'expose à le détruire. Il n'en reste pas moins que, comme le montre bien Jean Bodin, une des justifications fondamentales de l'absolutisme est la nécessité du changement. Si le prince est délié de l'obéissance aux lois, c'est pour pouvoir les faire ou les refaire. L'absolutisme prend la relève de ce que l'on a pu appeler le constitutionnalisme $^{29}$ (celui de Seyssel, par exemple), à partir du moment où il ne suffit plus de gouverner selon la loi, mais qu'il faut être capable de la faire. Le conformisme sceptique, l'accord avec la tradition n'ont plus lieu d'être ${ }^{30}$.

Or tel est bien, semble-t-il, le tournant que prend Gabriel Naudé. «Beaucoup, remarque-t-il, tiennent que le prince bien sage et avisé, doit non seulement commander selon les lois, mais encore aux lois même si la nécessité le requiert ${ }^{31}$. » Ses Considérations politiques sur les coups d'État enseignent comment, par des moyens extraordinaires et violents, on peut rétablir une autorité ébranlée. Renonçant à l' " universelle indifférence » de Charron, les « libertins érudits » se sont mis au service du pouvoir. Léonard Marandé fut secrétaire de Richelieu, quand Isaac La Peyrère était celui du prince de Condé, alors que François de La Mothe Le Vayer était précepteur

25. Essais, liv. I, chap. XXIII, « De la coutume et de ne changer aisément une loi reçue », in op. cit. supra n. 14, p. 120.

26. P. Charron, op. cit. supra n. 15, liv. II, chap. Il, p. 407.

27. Ibid., p. 412 .

28. Voir René PINTARD, Le Libertinage érudit dans la première moitié du xvif siècle, Paris, Boivin, 1943, qui soutient cette thèse souvent reprise.

29. Nannerl O. Keohane, Philosophy and the State in France. The Renaissance to the Enlightenment, Princeton, Princeton University Press, 1980.

30. Ce serait un anachronisme de confondre autoritarisme politique (l'Etat fort) et conservatisme. Par bien des aspects, l'absolutisme est en rupture avec les traditions et ouvert à la modernité.

31. Gabriel Naudé, Considérations politiques sur les coups d'État, rééd. Paris, Éditions de Paris, 1989, p. 76. 
du dauphin, futur Louis XIV. Mais c'est Naudé qui pratiqua le plus assidûment le conseil du prince : avant de revenir à Paris servir Richelieu (puis Mazarin), il fut, en Italie, celui d'un prélat romain, le cardinal Bagni. Dans un tel engagement, que devient le modèle sceptique de la liberté : celui de l'exercice privé du jugement hors de la presse du pouvoir?

Il semble qu'à se mêler de politique, le scepticisme ait perdu son nom. Dans les Considérations politiques sur les coups d'État, Louis Marin a trouvé " une théorie baroque de l'action politique ${ }^{32}$ " qui disparaît, selon lui, à l'âge classique. Montesquieu, en effet, s'en réjouit dans l'Esprit des lois :

"On a commencé à se guérir du machiavélisme, et on s'en guérira tous les jours. Il faut plus de modération dans les conseils. Ce qu'on appelait autrefois des coups d'Etat ne serait plus aujourd'hui, indépendamment de l'horreur, que des imprudences ${ }^{33}$.»

On aperçoit ainsi comment ce que l'on a nommé, en France, « machiavélisme » est avant tout la réception de conceptions politiques venues d'Italie, dont les libertins érudits furent les principaux passeurs.

Ces conceptions de la raison d'État, qui doivent sans doute plus à Giovanni Botero qu'à Nicolas Machiavel ${ }^{34}$, ont cependant en commun la place qu'elles accordent à l'intérêt. On sait à quel point ce concept, qui émerge chez Machiavel, va jouer un rôle central dans les théories modernes de la rationalité politique et, plus généralement, de la rationalité sociale ${ }^{35}$. On va, en effet, montrer que l'intérêt, c'est-à-dire le souci utilitaire de la conservation de l'existence, suffit à rendre compte des conduites, que ce soit celle d'un individu, ou d'une collectivité, tout particulièrement d'un État, sans qu'il soit nécessaire de supposer des motivations plus hautes (vertu) ou plus perverses (volonté délibérée de nuire aux autres). Cela tient à ce que l'intérêt est une raison non seulement suffisante, mais contraignante. Il oriente effectivement les actions et le grand succès de la catégorie d'intérêt, entendu plus étroitement comme souci d'un gain matériel plutôt que d'une satisfaction symbolique, est venu de ce que l'on a pu montrer que la répétition régulière des conduites intéressées pouvait venir à bout des excès passionnels, que l'intérêt avait vis-à-vis des passions traditionnelles (le pouvoir ou la guerre) des effets régulateurs et pacificateurs ${ }^{36}$. Voilà pourquoi

32. Louis Marin, Pour une théorie baroque de l'action politique, in G. NAUDE, op. cit. supra n. 31, p. 5-67.

33. Montesquieu, op. cit. supra n. 6, liv. XXI, chap. xx, t. II, p. 58.

34. Michel Senellart, Machiavélisme et raison d'État, Paris, Presses universitaires de France, 1989.

35. Albert O. HirschmanN, "Le concept d'intérêt ", in Vers une économie politique élargie, trad. franç., Paris, Minuit, 1986, p. 7-29. 1980.

36. ID., Les Passions et les intérêts, trad. franç., Paris, Presses universitaires de France, 
Montesquieu peut se réjouir. Si l'on a « commencé à se guérir du machiavélisme », ce n'est pas que l'on ait fait des progrès en vertu, mais que la répétition et la dispersion des échanges commerciaux mettent en place un réseau de relations qui échappent à l'emprise d'interventions ponctuelles et violentes. Les hommes ne sont pas meilleurs, ils sont forcés, par leur intérêt, de ne pas être trop méchants, les intérêts sont venus à bout des passions : « Il est heureux pour les hommes d'être dans une situation où, pendant que leurs passions leur inspirent la pensée d'être méchants, ils ont pourtant intérêt à ne pas l'être ${ }^{37}$."

Dans cette conception nouvelle, la rationalité politique ne s'appréhende pas comme une intention, ni comme un dessein anticipé, elle se constate dans les effets. Le moment sceptique a certainement joué un rôle important. La Mothe Le Vayer aborde l'étude de la politique avec un point de vue relativiste. Sans doute peut-on postuler l'existence d'une raison et d'une justice universelles, mais «cela n'empêche point qu'on ne doive déférer souvent, dans le sujet que nous traitons, à des raisons particulières qui varient selon les temps, les lieux et les personnes ${ }^{38}$ ». Si l'on peut parler de raison d'État, il ne faut entendre par là nulle généralité prêtant à science et à objectivité, mais la seule particularité d'une conduite individuelle : elle n'est « rien au fond qu'une pure considération d'intérêt ${ }^{39}$ ». S'en tenant aux faits, et refusant de les universaliser en devoirs, la démarche sceptique s'inscrit dans une approche de la politique qui a affaire aux intérêts du prince, plutôt qu'à ses vertus, et qui considère que le domaine de la politique est celui de la maîtrise des effets. De Blaise Pascal, mais aussi de Pierre Bayle, à Bernard Mandeville, de celui-ci à Adam Smith en passant par David Hume, on pourrait suivre les développements d'une réflexion où s'entrecroisent pessimisme religieux (augustinien) et relativisme sceptique pour donner une explication minimaliste de la rationalité des conduites, en termes d'intérêt. Un des aboutissements possibles de cette approche est la vision libérale selon laquelle il n'est pas nécessaire, mais nocif, d'orienter autoritairement des actions intéressées qui s'harmonisent d'elles-mêmes en un résultat positif.

Mais si le moment sceptique fait certainement partie de cette orientation de la réflexion politique, celle-ci le déborde de toutes parts, et fait intervenir d'autres composantes. Si l'on s'en tient aux " libertins érudits ", et à leur contribution à un éventuel modèle sceptique du politique, la question est plus circonscrite. Le débat, qui oppose Naudé et La Mothe Le Vayer,

37. Montesquieu, op. cit. supra n. 6, liv. XXI, chap. $\mathrm{xx}$, t. II, p. 58.

38. François de la Mothe Le Vayer, La Politique du prince, cité in Domenico Taranto, Pirronismo ed assolutismo nella Francia del' 600 . Studi sul pensiero politico dello scetticismo da Montaigne a Bayle (1580-1697), Milan, Franco Angeli, 1994, p. 126.

39. Petits Traités, lettre 53, in D. TARANTO, op. cit. supra n. 38, p. 128. 
porte avant tout sur le statut moral du prince, ce qui engage la façon dont se pondèrent, dans ces conceptions, composantes sceptiques et stoïciennes. Naudé pense pouvoir appliquer à la politique le modèle du sage, largement stoïcien, déjà élaboré par Charron. La mise en miroir du prince et de son conseiller (figure du sage) réalise un transfert qu'autorise l'élitisme repris de Charron : la liberté intérieure du sage, qui le met à l'écart des passions populaires, l'élève à des hauteurs où il peut rencontrer le prince, dans l'isolement d'un tête à tête. À l'abri du peuple « inconstant et variable, sans arrêt non plus que les vagues de la $\operatorname{mer}^{40}$ ", le prince et son conseiller recomposent, en une intimité confidentielle, l'espace sceptique qui soustrait la liberté de jugement aux diverses pressions extérieures. Mais c'est devenu le lieu même du pouvoir.

Du sage (retiré en son «dedans ») la politique du prince conserve sa caractéristique principale, le secret que n'annulent pas les Considérations politiques sur les coups d'État de Naudé. À l'inverse de Machiavel, dont on a pu dire qu'en publiant Le Prince il critiquait la tyrannie, car il en dévoilait les ressorts cachés, les Considérations sont une œuvre à diffusion confidentielle (douze exemplaires), qui mettent en scène, dans le redoublement de la fiction théorique, les arcana imperii, les renforçant sans les révéler. L'œuvre de Naudé est écrite en situation, celle du conseiller vis-àvis de son prince, et ne peut s'en abstraire, pour prétendre à l'universalité : elle n'échappe pas au relativisme sceptique.

C'est pourquoi la politique est une pratique, non une science. Elle est affaire de prudence, "vertu morale et politique, laquelle n'a autre but que de rechercher les divers biais, et les meilleures et plus faciles inventions de traiter et faire réussir les affaires que l'homme se propose ${ }^{41} »$. Gabriel Naudé en emprunte le modèle à Pierre Charron et à Justus Lipsius. C'est pour lui la capacité à s'adapter aux circonstances, et donc à faire face à la fortune. La prudence prend ainsi la place chez Naudé qui revenait, chez Machiavel, à la virtu, cette violence innovatrice, capable de résister à la fortune, et que Machiavel oppose à la prudence conservatrice. Sans doute la prudence n'est-elle pas, chez Naudé, exclusive de la violence du coup d'État, mais celle-ci, destinée à conserver l'État, restaure plus qu'elle n'instaure : point d'ordini nuovi mais seulement les « grands coups d'autorité », dont parle Montesquieu. Cela tient sans doute à ce que la réception française du machiavélisme tend à réinscrire, dans l'espace social et politique, une monarchie de longue histoire, mais ébranlée par la crise religieuse, plutôt qu'à favoriser l'accueil d'un prince nouveau. Du machiavélisme, cette réception prudentielle de la raison d'État retient l'inefficacité de la morale :

40. P. Charron, op. cit. supra n. 15, liv. I, chap. Lil, « Peuple ou vulgaire », p. 335.

41. G. NAUDE, op. cit. supra n. 31 , p. 88. 
la prudence politique s'accommode de la dissimulation et de tous moyens extraordinaires. Peut-on véritablement dire «sage » celui qui l'applique?

La Mothe Le Vayer ne le prétend pas. Il reprend l'idée de Montaigne selon laquelle « les âmes des Empereurs et des savetiers sont jetées à même moule $^{42}$ ». L'homme politique est soumis, comme tout un chacun, à ses passions, et seule la faiblesse caractéristique de l'esprit humain, peut lui faire croire qu'il a plus de clarté dans son jugement, plus de prévoyance dans la décision. Sans doute une telle approche conduit-elle à un autre régime de la rationalité politique (qui s'appréhende par les effets, non par les intentions). Mais la conséquence directe de la critique radicale que fait La Mothe Le Vayer de toute prétention à un savoir politique est autre. On peut suivre l'interprétation de Comparato : elle conduit plutôt à sortir du politique. L'objectif est de déconsidérer la grandeur affectée du politique :

« Mais s'il y a lieu où la petitesse de l'esprit de l'homme paraisse, c'est, à le bien prendre, en l'estime qu'il fait de tout ce qui regarde les dominations de la terre; de l'éclat et grandeur desquelles il est aisément ébloui, s'imaginant que tous leurs mouvements se font avec poids et mesure, et que les moindres choses y sont concertées avec une extraordinaire ratiocination. Et cependant il est certain qu'il n'y a rien de si faible que les principaux ressorts qui donnent le bransle à ces grandes machines, et rien de si imbécile que les liens qui tiennent les peuples par respect et par ignorance attachés à leurs destinées ${ }^{43}$. 》

Le sage s'est séparé du prince, il est seul au-dessus des autres. La place du sceptique est à l'écart du pouvoir.

\section{III. — LIBERTÉ DE PENSÉE}

Nous revenons ici à notre hypothèse de départ : celle d'une contribution originale du scepticisme à la liberté, que son alliance avec le catholicisme lui aurait donné les moyens de réaliser, dans une commune opposition au dogmatisme calviniste. Si l'hypothèse a quelque consistance, elle devrait impliquer que le mouvement sceptique - et tout particulièrement dans la période post-tridentine - a eu plus d'importance et d'ampleur dans les

42. Essais, liv. II, chap. XII, in op. cit. supra n. 14, p. 454.

43. F. DE LA MOTHE LE VAYER, De la politique, in ID., Les Neuf Dialogues faits à l' imitation des Anciens, 1630-1631, éd. André Pessec, Paris, Fayard (Corpus des auvres de philosophie en langue française), 1988, p. 412-413. L'idée que la force du pouvoir est une grandeur apparente ou empruntée, que le peuple obéit surtout par ignorance, se trouve à la fin du chapitre des Essais sur les cannibales. 
pays catholiques que dans les pays protestants. Dans la présentation qu'il fait des «érudits libertins», Robert Mandrou l'envisage globalement comme un phénomène européen, un réseau de correspondances, de voyages, de diffusion des échanges intellectuels et de la renommée, qui couvre toute l'Europe ${ }^{44}$. Il se forme ainsi un milieu unifié par «les liens qui les rapprochaient, ainsi que la communauté d'attitudes face à la vie qui réunissait des personnalités diverses douées de sensibilités semblables », selon Comparato ${ }^{45}$. Parmi ces traits communs se remarque l'utilisation que ces érudits font du champ sémantique de la liberté pour se définir : «ingénuité », « liberté », « générosité », « indépendance », « franchise » ${ }^{46}$. Le «catholicisme libéral » leur fournit-il cependant, mieux que la Réforme protestante, la possibilité d'affirmer leur « liberté philosophique »?

Si la distribution générale des «érudits libertins » est européenne, il semblerait qu'elle se concentre, de France en Italie, sur un axe Paris-Rome, alors que les petites capitales allemandes restaient attachées aux débats théologiques et protégeaient, en conséquence, d'abord des « maîtres-théologiens ». Pourtant, on trouve aussi des centres de réflexion érudite en pays protestants: Londres et l'Angleterre (dans le sillage de Francis Bacon), dans les Provinces Unies surtout; dans les villes, les universités, les écoles, foisonnent les « foyers de discussion et d'invention » vers lesquels les érudits libertins (Samuel Sorbière ou Simaise) se tournent autant que vers l'Italie ${ }^{47}$.

La liberté dont ils jouissent semble avoir plus directement rapport à la politique qu'à la religion. Martin Van Gelderen a montré comment la liberté de pensée et d'expression revendiquée durant la Révolte hollandaise, ne pouvait pas être envisagée comme une application directe de la liberté de conscience religieuse ${ }^{48}$. Retraçant la conception luthérienne de la conscience, depuis la syndérèse médiévale (cette parcelle de lumière naturelle qui, pour Guillaum d'Occam en particulier, subsiste en l'homme malgré la Chute), Van Gelderen fait voir que la liberté de conscience, telle que Martin Luther la défend, consiste en une attitude mentale qui permet d'être captivé par la vérité divine, de s'ouvrir à une vérité qui domine l'individu et à laquelle il se soumet. Luther, très significativement, remarque Van Gelderen, en appela à Worms, à sa conscience « captive de la parole de

44. Robert Mandrou, Histoire de la pensée européenne. T. III : Des humanistes aux hommes de science, $x v f^{e}$ et $x v f^{p}$ siècles, Paris, Seuil (Points/Histoire), 1973, chap. Iv, p. 142153.

45. I. Comparato, art. cit. supra n. 7, p. 190.

46. Ibid. Les termes sont pris dans la préface de F. DE LA MOTHE LE VAyER, op. cit. supra n. 43 .

47. R. MANDROU, op. cit. supra n. 44, p. 146.

48. Martin VAN Gelderen, « Liberté, droits et devoirs civiques dans l'Europe du xvi siècle $»$, in op. cit. supra n. 7, p. 115-142. 
Dieu " et non pas autonome ou indépendante en matière de salut ${ }^{49}$. Ce n'est que dans la foi, et dans la vraie foi, que le chrétien peut jouir de la liberté de conscience. Le vœu de l'Église réformée était de donner la possibilité à chaque chrétien de jouir d'une telle liberté, nullement de permettre aux mauvaises sectes de défendre leurs propres opinions, propres à séduire les âmes innocentes et à créer la discorde. Calvin, en défendant une liberté religieuse qu'il opposait à la licence, n'a fait que renforcer cette conception.

En Hollande, cependant, la liberté de conscience a été défendue comme une liberté d'opinion et d'expression. On y a vu le droit qu'avait tout individu de faire lui-même ses choix religieux : "Tout homme ordinaire a la permission et même le devoir de juger par lui-même de la vérité ou de la fausseté d'une doctrine ${ }^{50}$. » La liberté de conscience, alors, devenait un droit naturel qui impliquait nécessairement la liberté d'expression et de culte. Une telle inflexion par rapport au luthéranisme, qui fait de la liberté religieuse une des formes de la liberté d'expression ne s'explique que parce que celle-ci a été défendue, par ailleurs, sur d'autres terrains. Ce n'est pas la liberté religieuse qui rend possible d'autres formes de liberté, mais l'inverse : l'usage séculier de la liberté, qui s'est développé indépendamment, en accueille les manifestations religieuses :

"[...] c'est la pratique sociale, économique et politique de la République hollandaise, conclut Van Gelderen, qui créa un espace propice au développement de l'individualité. [...] La politique fédéraliste et républicaine, l'absence effective de censure donnaient aux citoyens tout loisir de s'engager dans de vastes débats publics passionnés ${ }^{51}$.»

C'est donc avant tout d'un modèle politique de la liberté, lié à des formes nouvelles du vivere civile (le débat plutôt que la participation au pouvoir) dont nous disposons ici.

La dimension proprement sceptique de l'érudition libertine peut nous fournir un autre modèle. L'accord du scepticisme et du fidéisme (que postule la thèse de Popkin) repose sur une conception qui est tout à l'opposé de la conception religieuse de la liberté de conscience, qui est celle d'une liberté captivée par la foi, et atteignant par là la vérité. La position sceptique implique au contraire la dualité de la foi et de l'opinion. La foi est mise à l'écart du débat sur la raison, et sur ses arguments, alors que ceux-là sont admis dans la multiplicité des opinions. Alors que dans la démarche religieuse, telle que la présente Luther, la liberté capture l'opinion - le

49. Ibid., p. 131.

50. Dirck V. COORnherdt, cité par M. VAN Gelderen, in art. cit. supra n. 48, p. 136.

51. M. VAN Gelderen, art. cit. supra n. 48, p. 142. 
jugement individuel - pour l'orienter vers la foi et l'élever, par là, à la vérité, la démarche sceptique scinde l'usage de la liberté : en même temps qu'elle soustrait la foi au doute, parce qu'elle n'en fait pas l'aboutissement d'un raisonnement, elle ouvre celui-ci à une prolifération argumentative qui donne un droit égal à toutes les opinions. Unité de la foi et multiplicité des opinions relèvent de deux domaines différents.

La démarche sceptique laisse donc pleine liberté à l'exercice du jugement, une liberté individuelle qui ne reste pas enfermée dans l'intériorité de la conscience, mais se pratique à plusieurs. La liberté que l'on a par rapport à ses propres opinions conduit à la tolérance à l'égard de celles des autres, comme on a vu Montaigne l'affirmer : celui qui sait suspendre son jugement est accueillant aux opinions des autres. La démarche sceptique propose donc bien un modèle de liberté, un modèle social de liberté. Mais c'est un modèle privé. Et c'est pourquoi il propose une solution différente de l'apologie républicaine de l'espace public.

De Jean-Pierre Vernant ${ }^{52}$ à Geoffrey E. Lloyd ${ }^{53}$, on a fort bien montré la convenance qu'il a pu y avoir entre le développement politique de la cité en Grèce et l'apparition concomitante de la « physique » grecque, des sages de Milet à Démocrite. La rationalité scientifique grecque est une rationalité argumentée, qui emprunte à la politique grecque ses modalités : la discussion entre citoyens égaux, la nécessité d'emporter la conviction par la capacité d'argumenter, l'aptitude à distinguer, dans le discours et l'usage du langage, entre le mythique et le rationnel.

Mais l'on a également montré que la révolution scientifique moderne qui s'accomplit de Galilée à Newton - parce que c'est une révolution contre le sens commun, implique une rupture décisive avec les modèles politiques de la rationalité grecque. Discipline d'observation, la science grecque fait appel au témoignage des sens. La science moderne ne s'appuie ni sur l'expérience immédiate (celle du soleil qui se lève), ni sur la seule argumentation rationnelle. Elle doit produire l'objet qu'elle connaît et authentifier les procédures de vérification. Cela suppose toute une culture de l'expérience : dans les lunettes astronomiques de Galilée, ses contemporains ne voyaient rien de ce qu'il prétendait y voir, et Kepler prend sa défense pour expliquer pourquoi on doit lui faire confiance ${ }^{54}$. La science moderne suppose la mise en place d'une communauté de savants, d'un public informé capable de témoigner des résultats d'une expérience et de la

52. Jean-Pierre Vernant, Mythe et pensée chez les Grecs, 2 vol., Paris, Maspero, 1965.

53. Geoffrey E. Lloyd, Pour en finir avec les mentalités, trad. franç., Paris, La Découverte, 1994.

54. Voir Catherine Chevalley, « Nature et loi dans la philosophie moderne », in Notions de philosophie, vol. I, op. cit. supra n. 13. 
refaire à son tour. La communauté scientifique se retire de la scène publique et $s^{\text {'impose ses propres règles }}{ }^{55}$.

On peut voir là comment le mouvement scientifique, au XvII ${ }^{e}$ siècle, a pu s'inscrire dans la suite du retrait humaniste de la scène publique, à la fin du $\mathrm{XvI}^{\mathrm{e}}$ siècle, et profiter de la formation d'espaces et d'institutions sociales organisant la communauté des lettrés qui avait accompagné ce retrait : les académies vont pouvoir, après les lettrés, être une base d'appui du travail des savants. Alors que la communauté des savants a besoin de réfléchir son existence propre, à l'écart des institutions publiques, la démarche sceptique lui offre un modèle social, d'un domaine privé de la réflexion commune, à l'écart de l'espace public de l'obéissance. Là où la tradition républicaine continue la tradition du débat public, le modèle sceptique convient mieux aux conditions nouvelles de l'exercice de l'enquête scientifique. Apporte$\mathrm{t}$-il, à cette vision sociale générale, une contribution plus spécifique?

Il nous semble que oui, sur le plan moral. Comme le scepticisme antique, le scepticisme moderne vise à définir une attitude morale et c'est d'ailleurs sur ce point qu'il sera mis en question : le sceptique peut-il vivre son scepticisme $^{56}$ ? Politiquement, non. L'échec d'une élaboration politique du scepticisme vient sans doute de l'impossibilité d'accorder un modèle moral, qui reste celui du sage au-dessus de la foule, avec une psychologie de l'intérêt qui fait du politique un homme comme les autres. Scientifiquement, oui. Le chapitre des Essais sur l'art de conférer ${ }^{57}$ offre une éthique de la vérité qui convient à la science moderne. C'est une éthique de la recherche, antidogmatique, d'une science « qui va enquêrant plutôt qu'instruisant », qui ne se donne pas de terme, mais reproduit sans cesse son propre mouvement. C'est une éthique de l'exercice partagé du jugement : "Le plus fructueux et naturel exercice de notre esprit, c'est à mon gré la conférence. " Elle fait plus qu'admettre la discussion, elle encourage la contradiction, l'associant aux valeurs de l'époque, celles d'une noblesse virile, le courage, la générosité, la liberté, l'égalité entre membres d'une même élite : « Nous fuyons à la correction, il s'y faudrait s'y présenter et produire, notamment quand elle vient par forme de conférence, non de rejance » (de leçon). Or une communauté de savants a besoin d'une telle éthique, pour régler ses rapports. Si l'on veut suivre la piste du « scepticisme modéré » qu'indique Popkin, celle d'une réponse «pragmatiste et

55. Steven Shapin et Simon Schaffer, Léviathan et la pompe à air. Hobbes et Boyle entre science et politique, trad. franç., Paris, La Découverte, 1993. Christian Licoppe, La Formation de la pratique scientifique. Le discours de l'expérience en France et en Angleterre (16301820), Paris, La Découverte, 1996.

56. Myles Burnyeat, « Can the sceptic live his scepticism? », in The Skeptical Tradition, éd. M. Burnyeat, Oxford, Clarendon Press, 1990, p. 20-53.

57. Voir Essais, liv. III, chap. vill, dans lequel les citations sans appel de notes qui suivent sont prises. 
positiviste » à la critique du dogmatisme, on pourrait bien, sur cette question, aboutir à nouveau à John Stuart Mill. Celui-ci montre en effet, dans On liberty (1859), comment, à partir du moment où l'on rejette l'idée dogmatique qu'une position peut être assimilée à la vérité, et où l'on admet qu'aucune affirmation ne peut prétendre à être plus qu'une opinion, le seul moyen de garantir un progrès continu vers la vérité est d'assurer la plus grande liberté du débat, d'exposer toutes les opinions à la contestation. En poussant même plus loin la filiation, on pourrait suivre la piste sceptique jusqu'à cette éthique de la clarté qui est le plus réel succès, selon Charles Larmore, de la philosophie analytique de langue anglaise : «[...] se rendre ouverte à la critique, chacun ayant en principe les moyens de vérifier si elle réussit ou non ${ }^{58}$. »

Qu'une telle éthique de la discussion ne préserve ni de l'universalisme dogmatique, ni du sectarisme hostile aux pensées dissidentes, c'est ce qu'une brève enquête sur l'utilitarisme ou la philosophie analytique contemporaine permettrait de vérifier aisément. Il faudrait donc rechercher si, en sus d'une éthique de la vérité, le scepticisme n'apportait pas également une certaine conception de la vérité, capable d'en garantir la quête sans fin. Cela conduirait à s'interroger sur une possible contribution sceptique à une conception probabiliste de la vérité : une conception qui n'en reste pas à l'alternative du vrai et du faux, mais considère qu'il peut y avoir des degrés du probable, une conception plus proche en cela du scepticisme de Carnéade que de celui de Pyrrhon; une conception qui, en outre, ouvre à la possibilité de la mathématisation. Enquête qu'il faudrait faire dans le domaine des sciences de la nature et dans la façon dont s'organise leur travail, sans doute. Mais il y a également une enquête à faire sur ce que l'on va appeler les «sciences morales». Il serait intéressant, par exemple, d'étudier de ce point de vue la critique que faisait Nicolas Fréret, à l'Académie des inscriptions, du "pyrrhonisme historique 59 ". Elle en remettait certes en cause les implications dogmatiques (si on ne sait pas tout, on ne sait rien), mais n'introduisait-elle pas également à une vision probabiliste de la vérité historique qui pouvait être comprise comme une réception positive de la critique sceptique?

Interroger le modèle libéral dont le scepticisme a pu être porteur, c'est ainsi passer de son versant religieux (le fidéisme) à son versant scientifique (le probabilisme). C'est par là même changer de type de pouvoir : il serait intéressant de savoir si, au compromis entre l'Église et les intellectuels que

58. Charles LaRmore, « Convictions philosophiques ", Philosophie. La philosophie continentale vue par la philosophie analytique, 35, 1992, p. 29.

59. Carlo Borghero, « Méthode historique et philosophie chez Fréret », Corpus, 29, 1995, p. 19-37. 
représente le fidéisme, correspond un compromis comparable entre la monarchie et ses savants. La monarchie française, aux XVII ${ }^{\mathrm{e}}$ et $\mathrm{XVIII}^{\mathrm{e}}$ siècles, a tenté d'appuyer son pouvoir politique sur le prestige des savoirs scientifiques (c'est de cette façon qu'est conduite l'action des jésuites français en Chine par exemple : conquérir la confiance de l'Empereur en lui montrant la supériorité des calculs astronomiques de la science européenne) : existet-il, entre le pouvoir politique et les communautés scientifiques, un intérêt commun au développement des savoirs et sur quoi repose-t-il?

Poser ces questions, c'est, nous semble-t-il, se placer dans le droit fil de la façon dont Popkin aborde l'histoire des idées, ou l'histoire intellectuelle. Elle lui permet de se passer des schémas unificateurs et réducteurs des visions téléologiques de l'histoire : la lutte front contre front de deux puissances opposées (la vision politique du monde : la lutte entre l'obscurantisme et les Lumières), et la permanence de visions monolithiques unifiées autour d'une prise de parti (la vision idéologique du monde : le matérialisme comme lutte contre la religion). À la place, on a l'étude de compromis ponctuels dans un contexte qu'ils visent et tendent à transformer, et l'étude des traditions, c'est-à-dire d'une succession des idées, dont la continuité est organisée par ceux-là mêmes qui les transmettent en les redéfinissant. Une telle approche nous paraît indispensable si l'on veut comprendre comment s'articulent, à l'époque moderne, savoirs et pouvoirs.

Elle nous semble également indispensable pour échapper au schématisme réducteur d'un affrontement manichéen entre un catholicisme obscurantisme et un protestantisme ami du savoir, par exemple, tout autant que pour tenter de comprendre comment l'enjeu philosophique qui conduit aux Lumières n'est pas seulement idéologique (« Écraser l'infâme ») mais, portant sur l'organisation des savoirs, épistémologique. Mais cela suppose que l'on développe une histoire intellectuelle capable de ne pas réduire les savoirs aux pouvoirs (dont ils seraient la simple expression instrumentale), capable aussi de maintenir la distinction des savoirs, et des formes de rationalité, tout en étudiant leurs rapports. Or c'est ce que permet une approche comme celle de Popkin.

Le grand mérite de Popkin est d'avoir mis en relation des domaines étudiés généralement séparément : l'histoire de la religion, celle de la philosophie, celle des sciences. Ce faisant, il ouvre sans doute à la possibilité de réduire un domaine à l'autre : on peut reprocher à Popkin de faire du scepticisme moderne un fidéisme, car cela conduit à ne donner au scepticisme qu'un rôle instrumental, à en méconnaître la dimension proprement philosophique, qui est à elle-même sa propre fin, donc à rabattre le philosophique sur le religieux ${ }^{60}$. Peut-être. On ne peut cependant oublier que

60. Voir, dans ce numéro, la contribution de Sylvia GiocanTI, « Histoire du fidéisme, histoire du scepticisme? », p. 193-210. 
c'est à Popkin que revient le mérite d'avoir fait apparaître l'importance du scepticisme dans l'histoire de la philosophie, d'en avoir fait un des grands fils conducteurs de la réflexion sur la révolution scientifique moderne, là où, traditionnellement (particulièrement en France), on ne lui accordait qu'un rôle marginal. Il serait tout à fait regrettable que le scepticisme une fois réinstallé - grâce à des travaux comme ceux de Popkin - à l'intérieur de l'histoire de la philosophie, celle-ci ne se préoccupât plus que de sa dimension proprement philosophique, oubliant que celle-ci n'avait pu apparaître que parce que la philosophie avait été mise en rapport avec d'autres domaines de la rationalité.

Tenter de continuer dans les voies ouvertes par Popkin (ce qui est le meilleur hommage que l'on puisse lui rendre, et la meilleure critique que l'on puisse en faire), ce n'est pas seulement étudier, dans l'un des domaines de l'histoire intellectuelle que son enquête parcourt (religion, science, philosophie), les traits nouveaux qu'il a fait apparaître. Cela requiert que l'on maintienne la circulation entre les trois domaines, que l'on continue à les rapporter les uns aux autres, en évitant deux pièges : le morcellement particulariste, l'universalisme uniformisant.

C'est l'avantage qu'il y a à prendre en compte la dimension politique de la thèse de Popkin. Tant qu'on en reste à la vision religieuse du fidéisme, la réduction du scepticisme (philosophique) au fidéisme (religieux) est possible. Étudier le compromis qui fait se rencontrer des intellectuels et des clercs sur un intérêt commun, ce n'est plus affirmer une unification réductrice, mais voir comment s'articulent des intérêts et des domaines qui restent distincts. Les sceptiques ont pu appuyer des positions religieuses, ils ont pu accepter que leur propre démarche soit réinscrite comme fidéisme dans le champ religieux : cela ne signifie ni que leur démarche se réduise au fidéisme, ni qu'elle se limite à son seul développement philosophique, puisque, en même temps, elle leur permet d'avoir rapport aux transformations scientifiques contemporaines. Alliances et compromis : cela permet de prendre en compte la pluralité des intérêts. Cela permet également d'éviter l'inversion schématique qui, de la vision traditionnelle de « libertins " antireligieux, fait des chrétiens sincères : ce que cherchaient peut-être les «libertins érudits», tels que les présente Popkin, c'est un espace de liberté pour vivre une foi qu'ils entendaient ne pas confondre avec la superstition. Ils pouvaient donc critiquer celle-ci (et les arguments peuvent être réutilisés dans des polémiques plus directement antireligieuses), sans cesser d'en faire partie : la foi, alors, est peut-être plus une façon de vivre - qui n'exclut pas les sentiments - qu'une position intellectuelle (forcément dogmatique). Elle est alors compatible avec une mise en cause de la rationalité qui se fait dans la constante, et exigeante, pratique de l'argumentation rationnelle. 
Étudier la dimension politique du livre de Popkin conduit ainsi à comprendre à la fois la pluralité des domaines et les médiations qui les rapportent les uns aux autres. C'est pourquoi, me semble-t-il, le livre de Popkin est un grand livre de philosophie politique.

Catherine LARRÈRE (janvier 1997). 